PFC/JA-88-29

CONDITIONS FOR ABSOLUTE INSTABILITY

IN THE CYCLOTRON RESONANCE MASER

John A. Davies*
Plasma Fusion Center
Massachusetts Institute of Technology
Cambridge, MA 02139
July, 1988

*Permanent address: Clark University, Worcester, MA 01610 


\title{
CONDITIONS FOR ABSOLUTE INSTABILITY IN THE CYCLOTRON RESONANCE MASER
}

\author{
John A. Davies* \\ Plasma Fusion Center \\ Massachusetts Institute of Technology \\ Cambridge, MA 02193
}

\begin{abstract}
This paper presents exact analytic conditions for absolute instability in the cyclotron resonance maser for the case of a cold beam, lossless circular waveguide, and infinite interaction length in the axial direction. The conditions are expressed in terms of the parallel beam velocity, the applied magnetic field strength, and the strength of the coupling between the beam and waveguide modes. The results are applicable to both the gyrotron and cyclotron autoresonance maser operating regimes.
\end{abstract}

* Permanent address: Clark University, Worcester, MA 01610 


\section{INTRODUCTION}

The cyclotron resonance maser (CRM) employs the electron cyclotron maser instability to generate high-power radiation in the centimeter to submillimeter wavelength regimes. ${ }^{1-7}$ The two principle operating regimes of the CRM are that in which $v_{p h} \gg c$ and that in which $v_{p h} \simeq c$. Here $v_{p h}$ is the phase velocity of electromagnetic radiation in the direction of the applied magnetic field. If $v_{p h} \gg c$, then the device is referred to as a gyrotron, whereas a device for which $v_{p h} \simeq c$ is called a cyclotron autoresonance maser (CARM). The CARM is an alternative form of free electron laser for radiation in the millimeter and submillimeter wavelength regimes. CARM oscillators and amplifiers are presently attracting attention as a promising source of high-power, millimeter-wavelength radiation for electron cyclotron resonant heating (ECRH) of fusion plasmas. The large Doppler upshift and efficiency of the CARM interaction allows the generation of high powers at the high frequencies (280-560 GHZ) required for ECRH on the next generation of tokamaks. One of the key issues which remains, and is in part addressed by this paper, is the generation of unwanted secondary modes in both the gyrotron and CARM regimes. ${ }^{8,9}$

In a CRM, the coupling of the electron beam mode to the waveguide mode produces instabilities which may be convective at smaller values of the beam current but which become absolute at larger values of the beam current. An instability is convective if the response of the system to a perturbation propagates away from the point of origin. An instability is absolute if the response grows in both directions about the point of origin. In the CRM, absolute instability is produced by the coupling of the beam mode to a backward traveling waveguide mode. The presence of an absolute instability can have deleterious effects. For example, an amplifier designed without accounting for the possible presence of an absolute instability might operate as an oscillator rather than an amplifier.

Absolute instability in the CRM is of greater concern than absolute instability in a free electron laser (FEL) employing a transverse wiggler field. It is not difficult to understand why this statement is true in the instance of the CARM. In the cases of both the CARM and the FEL two unstable modes are present. For the CARM, these modes

correspond to the two intersections of the uncoupled waveguide-mode dispersion relation 
with the uncoupled beam-mode dispersion relation on a plot of $\omega$ vs. $k$. For the FEL, the corresponding dispersion relations are the uncoupled waveguide-mode dispersion relation and the uncoupled dispersion relation for Doppler shifted plasma oscillations. We refer to these two instabilities as the downshifted instability (smaller $k$ ) and the upshifted instability (larger $k$ ). For either the CARM or the FEL, the upshifted instability is convective and is responsible for the generation of the desired radiation. The downshifted instability may be absolute. Plots of $\operatorname{Im}(\omega)$ vs. real $k$ for both the free electron laser and the CARM show two maxima. These are the downshifted maximum and the upshifted maximum. The temporal growth rate of the upshifted instability equals the height of the upshifted maximum. The temporal growth rate of an absolute instability lies between zero and the value of the downshifted maximum. ${ }^{10}$ Typically, in the case of the cold-beam free electron laser, the height of the upshifted maximum is much greater than the height of the downshifted maximum. These heights are comparable only for very low energy systems. On the other hand, in the case of the cold-beam CARM with a lossless waveguide, the height of the downshifted maximum exceeds that of the upshifted maximum.

In this paper, we derive exact, analytic conditions for absolute instability in a CRM employing a lossless circular waveguide and a cold relativistic electron beam. The conditions are presented in Sec. 2 in terms of three parameters. These are $\epsilon$ (the coupling constant), $\beta_{\|}$(the longitudinal electron velocity), and $s b=s \Omega_{c} / \omega_{c}$, where $s$ is the waveguidemode harmonic, $b$ is the normalized magnetic field, $\Omega_{c}$ is the cyclotron frequency, and $\omega_{c}$ is the waveguide-mode cutoff frequency. Over most of the useful range of CRM parameters, the condition for absolute instability is given in Eqs. (3)-(5). However, a different condition, given in Eqs. (3) and (9)-(11), must be applied in regions of the parameter space where $\beta_{\|}$is small. Equation (7) or (8) is used to determine which of the two conditions is applicable.

Our work is an extension of previous work of Lau et al. presented in Ref. 8. In Ref. 8, conditions for absolute instability are obtained for the CRM by assuming that the grazing condition $\left[b=b_{0}=\left(1-\beta_{\|}^{2}\right)^{\frac{1}{2}}\right]$ is approximately satisfied. Therefore, these results are applicable to the gyrotron and the gyro-traveling wave amplifier but not to the CARM. Also, we show in this paper that there is a restriction on the range of $\beta_{\|}$to which the 
results of Ref. 8 are applicable. Our results are not subject to the above restrictions and are applicable to both the gyrotron and the CARM regimes.

The derivation of the conditions given in Sec. 2 is presented in Sec. 3 and is based on the pinch-point theory of Briggs and Bers. ${ }^{11,12}$ Our treatment depends in part on analytical results and in part on observations of numerically computed images of the lowered Laplace contour in the complex $k$-plane. Because some of our inferences are based directly upon such observations, the derivation is not thoroughly rigorous. However, the results of Sec. 2 have been verified by extensive numerical computations of pinch-point coordinates as functions of the system parameters. 


\section{EXACT CONDITIONS FOR ABSOLUTE INSTABILITY}

We consider a CRM configuration in which a cold, annular electron beam propagates along an axial magnetic field $B_{0}$ inside a lossless, circular waveguide of radius $r_{w}$. We assume a zero electron guiding-center spread, with all guiding centers located on a cylinder of radius $r_{b}$ about the waveguide axis. For this configuration, the dispersion relation describing the interaction of the electron-beam mode and the $\mathrm{TE}_{\mathrm{mn}}$ waveguide mode is ${ }^{4,8}$

$$
D(\hat{k}, \hat{\omega})=\left(\hat{\omega}^{2}-\hat{k}^{2}-1\right)\left(\hat{\omega}-\beta_{\|} \hat{k}-s b\right)^{2}+\epsilon=0 .
$$

In the above equation, the dimensionless frequency $\hat{\omega}$ and wavenumber $\hat{k}$ are given by $\hat{\omega}=\omega / \omega_{c}$ and $\hat{k}=k / k_{m n}$, where $\omega_{c}=c k_{m n}$ is the cutoff frequency for the $\mathrm{TE}_{\mathrm{mn}}$ waveguide mode. The quantity $k_{m n}$ is given by $k_{m n}=\nu_{m n} / r_{w}$, where $\nu_{m n}$ is the $n$-th zero of the first derivative of the Bessel function $J_{m}$. Other quantities are the harmonic $s$ of the waveguide mode, the longitudinal electron velocity $\beta_{\|}$, and the normalized magnetic field $b=\Omega_{c} / \omega_{c}$. The quantity $\Omega_{c}=e B_{0} / \gamma_{0} m c$ is the relativistic cyclotron frequency for electrons of energy $\gamma_{0} m c^{2}$. The term $\epsilon$ couples the waveguide $\left(\hat{\omega}^{2}-\hat{k}^{2}-1\right)$ and beam $\left(\hat{\omega}-\beta_{\|} \hat{k}-s b\right)$ dielectric functions, which appear as factors in the left-hand term in Eq. (1). At instability, $\hat{\omega}-\beta_{\|} \hat{k}-s b \simeq 0$. As a consequence, a good approximation for the coupling term $\epsilon$ is ${ }^{8,13}$

$$
\epsilon=\frac{4 \beta_{\perp}^{2}}{\gamma_{0} \beta_{\|}} \frac{\left[J_{s-m}\left(k_{m n} r_{b}\right) J_{s}^{\prime}\left(k_{m n} r_{L}\right)\right]^{2}}{\left(\nu_{m n}^{2}-m^{2}\right) J_{m}^{2}\left(\nu_{m n}\right)} \frac{I}{I_{A}} .
$$

In the above equation, $\beta_{\perp}$ is the transverse electron velocity, and $r_{L}=c \beta_{\perp} / \Omega_{c}$ is the electron Larmor radius. The beam current is given by $I$, and $I_{A}=m c^{3} / e=17 k A$.

Equation (1) is fully specified by the three parameters $\beta_{\|}, s b$, and $\epsilon$. Consequently, absolute instability will be represented by regions in a three-dimensional parameter space. In the remainder of this section, we state and discuss exact conditions for the existence of absolute instability in a cold-beam CRM with a lossless waveguide. The derivation of the conditions is given in Sec. 3 .

As $s b$ approaches one from below, the CRM mode becomes absolutely unstable for all $\beta_{\|}$and $\epsilon>0$. For the case of $s b<1$, the mode is absolutely unstable if

$$
\epsilon>\epsilon_{c}
$$


where $\epsilon_{c}$ is the critical coupling constant for absolute instability. Over most of the useful range of values of $\beta_{\|}$and $s b$, it is given by

$$
\epsilon_{c}=27 \beta_{\|}^{2} \hat{k}_{s}^{4}
$$

The quantity $\hat{k}_{s}$ is the wave number of the absolutely unstable mode at the onset of absolute instability. It is given by

$$
\hat{k}_{s}=\frac{1}{2}\left(1+8 \beta_{\|}^{2}\right)^{-1}\left\{-4 \beta_{\|} s b+\left[16 \beta_{\|}^{2} s^{2} b^{2}+2\left(1+8 \beta_{\|}^{2}\right)\left(1-s^{2} b^{2}\right)\right]^{\frac{1}{2}}\right\} .
$$

The corresponding frequency is

$$
\hat{\omega}_{s}=4 \beta_{\|} \hat{k}_{s}+s b
$$

However, there is a restriction on the use of Eqs. (4) and (5) for the calculation of $\epsilon_{c}$. These equations are applicable if and only if

$$
\left.\frac{\partial D(\hat{k}, \hat{\omega})}{\partial \hat{\omega}}\right|_{\hat{k}_{s}, \hat{\omega}_{s}}>0
$$

Substituting Eqs. (1) and (6) into Eq. (7), we reduce the restriction in Eq. (7) to

$$
\hat{\omega}_{s}-\frac{\hat{k}_{s}}{\beta_{\|}}>0
$$

If the condition in Eq. (7) or (8) is not obeyed, then the critical coupling constant to be used in Eq. (3) is given by

$$
\epsilon_{c}=\frac{\hat{k}_{s}^{\prime}}{\beta_{\|}^{4}}\left(b_{0}^{2} \hat{k}_{s}^{\prime}-\beta_{\|} s b\right)^{3}
$$

where the wave number $\hat{k}_{s}^{\prime}$ of the absolutely unstable mode at the onset of absolute instability is now given by

$$
\hat{k}_{s}^{\prime}=\frac{\beta_{\|}}{4 b_{0}^{2}}\left[s b+\left(s^{2} b^{2}+8 b_{0}^{2}\right)^{\frac{1}{2}}\right] .
$$

The corresponding frequency $\hat{\omega}_{3}^{\prime}$ is given by

$$
\hat{\omega}_{s}^{\prime}=\frac{\hat{k}_{s}^{\prime}}{\beta_{\|}} .
$$


In Eq. (10), we have introduced $b_{0}$, the grazing value of $s b$. It is given by

$$
b_{0}=\left(1-\beta_{\|}^{2}\right)^{\frac{1}{2}}
$$

At grazing the uncoupled dispersion curves ( $\hat{\omega}$ vs. real $\hat{k})$ for the waveguide and beam modes are tangential.

In Fig. 1 , we present plots of $\epsilon_{c}$ vs. $\beta_{\|}$for several values of $s b<1$ shown against the curves. A curve $(A B)$ of $\hat{\omega}_{s}=\hat{k}_{s} / \beta_{\|}$[obtained from Eqs. (5) and (6)] is also plotted in Fig. 1. The condition in Eq. (8) is satisfied for all parameter points to the right of this curve. Consequently, plots of $\epsilon_{c}$ to the right of $A B$ are obtained from Eqs. (4)-(6), and those to the left are obtained from Eqs. (9)-(11). A CRM mode will be absolutely unstable if its parameter point lies above that $\epsilon_{c}$ curve which corresponds to its value of $s b$. Notice that all of the large $\beta_{\|}$parameter region applicable to the CARM and much of the small $\beta_{\|}$parameter region applicable to the gyrotron lie to the right of $A B$. Figure 1 includes the limiting curve of $\epsilon_{c}$ for the case of $s b=0$. All modes whose parameter points lie above this curve are absolutely unstable. We remark that this curve does not imply that a CRM can be absolutely unstable when the applied axial magnetic field $B_{0}$ is zero. For, in this case, the coupling constant $\epsilon$ vanishes.

Regions of absolute instability in Fig. 1 can also be computed numerically. Beginning at some large value of $\epsilon$, we reduce $\epsilon$ in magnitude until $\operatorname{Im}(\hat{\omega})$ at the pinch point, representing absolute instability, first reaches zero. (See Sec. 3 for a brief discussion of pinch points.) Results of such computations agree exactly with the plots in Fig. 1.

Often the critical current above which the system will be absolutely unstable is of interest. We see from Eq. (2) that this current is given by

$$
I_{c}=\frac{\gamma_{0} \beta_{\|}}{4 \beta_{\perp}^{2}} \frac{\left(\nu_{m n}^{2}-m^{2}\right) J_{m}^{2}\left(\nu_{m n}\right) I_{A} \epsilon_{c}}{\left[J_{s-m}\left(k_{m n} r_{b}\right) J_{s}^{\prime}\left(k_{m n} r_{L}\right)\right]^{2}}
$$

where $\epsilon_{c}$ is given by Eqs. (4) and (5), if the condition in Eq. (8) is satisfied. Otherwise, $\epsilon_{c}$ is given by Eqs. (9)-(11). 


\section{DERIVATION OF EXACT CONDITIONS}

We briefly summarize the conditions for absolute instability as derived by Briggs and Bers. ${ }^{11,12}$ The response $\psi(z, t)$ of a one-dimensional system to a delta-function disturbance in space and time is given by

$$
\psi(z, t)=\int_{L} d \omega e^{-i \omega t} \int_{F} d k \frac{e^{i k z}}{D(k, \omega)}
$$

Here, $L$ is the inverse Laplace contour (in the complex $\omega$-plane) taken above all zeros of the dielectric function $D(k, \omega)$, and $F$ is the inverse Fourier contour (in the complex $k$-plane) taken along the real $k$-axis. Absolute instability is detected by lowering the $L$-contour toward the real $\omega$-axis and noting the behavior of the images of the $L$-contour in the $k$ plane obtained from the dispersion relation $D(k, \omega)=0$. Initially none of the images cross the real $k$-axis. Convective instabilities occur if images cross this axis as the $L$-contour is lowered. However, if an image from above the real $k$-axis merges with one from below to form a saddle point of $\omega(k)$ (before the $L$-contour reaches the real $\omega$-axis), then the instability is absolute. Such a saddle point is called a pinch point. The complex wave number $k_{s}$ and complex frequency $\omega_{s}$ of the absolutely unstable mode are given by $k$ and $\omega$ at the pinch point.

The conditions for a saddle point in $\omega(k)$ are that

$$
D(k, \omega)=0
$$

and

$$
\frac{\partial D(k, \omega)}{\partial k}=0
$$

Applying Eq. (15) to the dispersion relation in Eq. (1), we obtain the saddle point condition

$$
\hat{k}_{s}\left(\hat{\omega}_{s}-\beta_{\|} \hat{k}_{s}-s b\right)+\beta_{\|}\left(\hat{\omega}_{s}^{2}-\hat{k}_{s}^{2}-1\right)=0
$$

Then, substituting Eq. (16) into Eq. (1), we obtain a second independent saddle point condition

$$
\beta_{\| \epsilon} \epsilon=\hat{k}_{s}\left(\hat{\omega}_{s}-\beta_{\|} \hat{k}_{s}-s b\right)^{3}
$$


We obtain the critical value of the coupling term $\left[\epsilon_{c}\right.$ in Eq. (3)] by using a combination of analytic and numerical methods. Much of our derivation is an extension of that given in Ref. 8. At the onset of absolute instability, the value of $\operatorname{Im}\left(\hat{\omega}_{s}\right)$ at the pinch point is zero. A very important consequence of this fact (proved in Ref. 8) is that [for the dispersion relation in Eq. (1)] the pinch point lies on the real $\hat{k}$-axis at the onset of absolute instability. Consequently, as $\epsilon$ is increased to $\epsilon_{c}$, a saddle point forms on the real $\hat{k}$-axis. An indication of this formation is the merging of two or more real roots of the dispersion relation on the real $\hat{k}$-axis. Therefore, we investigate the merging of roots of the dispersion relation on the real $\hat{k}$-axis with increasing $\epsilon$. In order to investigate this merging graphically, we rewrite the dispersion relation in Eq. (1) in the form

$$
\mathcal{D}(\hat{k}, \hat{\omega})=\left[\hat{k}^{2}-\left(\hat{\omega}^{2}-1\right)\right]\left[\hat{k}-\frac{(\hat{\omega}-s b)}{\beta_{\|}}\right]^{2}=\frac{\epsilon}{\beta_{\|}^{2}} .
$$

Schematic plots of $\mathcal{D}(\hat{k}, \hat{\omega})$ vs. real $\hat{k}$ for several of values of $\hat{\omega}$ are shown in Fig. 2. The plots are for the case of $s b<b_{0}$, where $b_{0}$ is the grazing value of $s b$ [Eq. (12)]. For purposes of the present analysis, plots for the case of $b_{0}<s b<1$ do not differ significantly from those for $s b<b_{0}$. (Similar graphs are discussed in Ref. 8.) For a given $\epsilon$, real roots of Eq. (1) or (18) occur at the intersection of the plots with the horizontal line $\mathcal{D}(\hat{k}, \hat{\omega})=\epsilon / \beta_{\|}^{2}$.

If $\epsilon=0$ and $\hat{\omega} \geq 1$, then Eq. (18) has four real roots. These are the two beam-mode roots $\hat{k}=(\hat{\omega}-s b)^{2} / \beta_{\|}$, the forward-traveling waveguide-mode root $\hat{k}=\left(\hat{\omega}^{2}-1\right)^{\frac{1}{2}}$, and the backward-traveling waveguide-mode root $\hat{k}=-\left(\hat{\omega}^{2}-1\right)^{\frac{1}{2}}$. By regarding $\hat{k}(\hat{\omega}, \epsilon)$ as an analytic function of $\hat{\omega}$ and a continous function of the real parameter $\epsilon$, we can follow the real roots arising from the waveguide and beam modes as $\epsilon$ is increased above zero in Fig. 2. Absolute instability requires the merging of a root which is below the real $\hat{k}$-axis for large $\operatorname{Im}(\hat{\omega})$ with one which is above the real $\hat{k}$-axis for large $\operatorname{Im}(\hat{\omega})$. Clearly, $\operatorname{Im}(\hat{k})$ of a root arising from the backward-traveling waveguide mode goes to $-\infty$ as $\operatorname{Im}(\hat{\omega})$ goes from zero to $+\infty$. Moreover, $\operatorname{Im}(\hat{k})$ goes to $+\infty$ as $\operatorname{Im}(\hat{\omega})$ goes from zero to $+\infty$ for roots arising from the forward-traveling waveguide mode and the beam modes. Consequently, on physical grounds, we look for the merging of a real root arising from the backward-traveling waveguide mode (which comes from below the real $\hat{k}$-axis) with a real root arising from a beam mode (which comes from above the real $\hat{k}$-axis). Such merging is seen to occur only 
in Figs. 2d and 2g. An examination of these figures shows that this merging takes place at points of inflection, where three real roots merge. (These are the two roots arising from the waveguide modes and one root arising from a beam mode.) This merging represents the formation of a third-order saddle point on the real $\hat{k}$-axis in the complex $\hat{k}$-plane. At such a saddle point,

$$
\frac{\partial^{2} \mathcal{D}(\hat{k}, \hat{\omega})}{\partial \hat{k}^{2}}=\frac{\partial^{2} D(\hat{k}, \hat{\omega})}{\partial \hat{k}^{2}}=0
$$

Written out explicitly, Eq. (19) is

$$
-\left(\hat{\omega}-\beta_{\|} \hat{k}-s b\right)^{2}+4 \beta_{\|} \hat{k}\left(\hat{\omega}-\beta_{\|} \hat{k}-s b\right)+\beta_{\|}^{2}\left(\hat{\omega}^{2}-\hat{k}^{2}-1\right)=0 .
$$

The coordinates of the third-order saddle points are obtained by solving Eqs. (20) and (16) simultaneously for $\hat{k}_{s}$ and $\hat{\omega}_{s}$. It is not necessary to make the assumption of Ref. 8 that the system is near grazing [i.e., that $s b \simeq b_{0}=\left(1-\beta_{\|}^{2}\right)^{\frac{1}{2}}$ ]. We obtain $\hat{\omega}_{s}=4 \beta_{\|} \hat{k}_{s}+s b$ [Eq. (6)] together with

$$
\hat{k}_{s}=\frac{1}{2}\left(1+8 \beta_{\|}^{2}\right)^{-1}\left\{-4 \beta_{\|} s b \pm\left[16 \beta_{\|}^{2} s^{2} b^{2}+2\left(1+8 \beta_{\|}^{2}\right)\left(1-s^{2} b^{2}\right)\right]^{\frac{1}{2}}\right\} .
$$

Tentatively, we identify the critical coupling constant $\epsilon_{c}$ with the smaller of the two values of $\epsilon$ associated with the inflection points in Figs. $2 \mathrm{~d}$ and $2 \mathrm{~g}$. Then, the critical coupling term given in Eq. (4) (i.e., $\epsilon_{c}=27 \beta_{\|}^{2} \hat{k}_{s}^{4}$ ) follows from Eq. (6) and (17). Equation (21) contains two solutions corresponding to the two inflection points. The solution with the positive square root (corresponding to the inflection point shown in Fig. 2d) produces a smaller value of $\epsilon_{c}$ in Eq. (4) than that with the negative square root. Therefore this solution is presented in Eq. (5). Equations (4)-(6) reduce to Eqs. (7)-(9) of Ref. 8 when $s b=b_{0}$.

Our tentative identification of the critical coupling constant as the value of $\epsilon$ associated with the point of inflection in Fig. $2 \mathrm{~d}$ is not valid over the total range of $\beta_{\|}$. The formation of the third-order saddle point on the real $\hat{k}$-axis represents the onset of absolute instability only if an increase in $\epsilon$ above the tentative value of $\epsilon_{c}$ given by Eq. (4) produces a saddle point with a positive $\operatorname{Im}(\hat{\omega})$, i.e., a pinch point. Whether or not formation of a pinch point occurs depends upon the detailed behavior of curves of $\mathcal{D}(\hat{k}, \hat{\omega})$ vs. $\hat{k}$ in the neighborhood 
of the inflection point in Fig. 2d. Figures $3 \mathrm{a}$ and $3 \mathrm{~b}$ are examples of plots of $\mathcal{D}(\hat{k}, \hat{\omega})$ vs. real $\hat{k}$ near the inflection point for various values of real $\hat{\omega}$. The parameter $s b=0.6$ in both figures, whereas the respective values of $\beta_{\|}$are 0.8 and 0.05 . In Fig. 3a, $\mathcal{D}(\hat{k}, \hat{\omega})$ is increasing with decreasing $\hat{\omega}$ in the neighborhood of the inflection point. Consequently, the maxima and minima which merge to form the inflection point lie below the inflection point. (See Figs. 2a-2d.) It is clear from Fig. 3a that increasing $\epsilon$ above $\epsilon_{c}$ causes $\hat{\omega}$, to become complex. In fact, numerical computations show that the third-order saddle point splits into two second-order saddle points in the complex $\hat{k}$-plane, one of which is a pinch point. On the other hand, $\mathcal{D}(\hat{k}, \hat{\omega})$ is decreasing with decreasing $\hat{\omega}$ in Fig. 3b. Consequently, the maxima and minima lie above the inflection point. We see from the figure that increasing $\epsilon$ above $\epsilon_{c}$ causes the third-order saddle point to split into two second-order saddle points, and that both lie on the real $\hat{k}$-axis with $\operatorname{Im}(\hat{\omega})=0$. Therefore, no pinch point is formed.

Consequently, Eqs. (4) and (5) can be used to determine the critical coupling constant if and only if $\partial \mathcal{D}(\hat{k}, \hat{\omega}) / \partial \hat{\omega}<0$ at the inflection point in Fig. 2d. The condition in Eq. (7) follows from the fact that $\partial \mathcal{D}(\hat{k}, \hat{\omega}) / \partial \hat{\omega}$ and $\partial D(\hat{k}, \hat{\omega}) / \partial \hat{\omega}$ have opposite signs.

To illustrate and expand the above discussion, we present numerically computed plots of images of the $L$-contour in the complex $\hat{k}$-plane. The contours in Fig. 4 are obtained from the parameters of Fig. 3a (i.e., $s b=0.6$ and $\beta_{\|}=0.8$ ). In Fig. $4 a, \epsilon<\epsilon_{c}$ and there are two saddle points on the real $\hat{k}$-axis with $\operatorname{Im}(\hat{\omega})=0$. When $\epsilon=\epsilon_{c}$, these saddle points merge to form a single third-order saddle point with $\operatorname{Im}(\hat{\omega})=0$, as is shown in Fig. $4 \mathrm{~b}$. Referring to Fig. 4c, we see that increasing $\epsilon$ above $\epsilon_{c}$ causes the saddle point to split into two second-order saddle points, one above and one below the real $\hat{k}$-axis. The saddle point with $\operatorname{Im}(\hat{\omega})>0$ lies below the real $\hat{k}$-axis. This saddle point is a pinch point, because additional contour plots (not included here) confirm that this saddle point is formed by the merging of an $L$-contour image from above the real $\hat{k}$-axis with one from below. This result is expected, because three of the images of the original Laplace contour lie above the real $\hat{k}$-axis and only one lies below. [This fact is proved by letting $\operatorname{Im}(\hat{\omega})$ approach infinity in Eq. (1).]

Numerically computed contours for the parameters of Fig. $3 \mathrm{~b}\left(s b=0.6\right.$ and $\left.\beta_{\|}=0.05\right)$ are shown in Fig. 5. Fig. 5a shows that if $\epsilon<\epsilon_{c}$, then there are two second-order saddle 
points in the complex $\hat{k}$-plane, and that the saddle point with $\operatorname{Im}(\hat{\omega})>0$ lies in the upper-half $\hat{k}$-plane. Numerically, we find that this saddle point is formed by the merging of two branches whose original Laplace contour images are in the upper-half $\hat{k}$-plane. Consequently, this saddle point is not a pinch point. In Fig. 5b, we see that increasing $\epsilon$ to $\epsilon_{c}$ causes the two saddle points to merge into a single third-order saddle point on the real $\hat{k}$-axis with $\operatorname{Im}(\hat{\omega})=0$. Reference to Fig. $5 \mathrm{c}$ shows that a further increase in $\epsilon$ splits this third-order saddle point into two second-order saddle points, both of which are on the real $\hat{k}$-axis with $\operatorname{Im}(\hat{\omega})=0$. Consequently, none of the saddle points shown in Fig. 5 are pinch points.

Next we determine the critical coupling constant for the case where Eq. (7) is not satisfied [i.e., the case where $\partial D(\hat{k}, \hat{\omega}) / \partial \hat{\omega}<0]$. Since the inflection point in Fig. $2 \mathrm{~b}$ does not represent the onset of absolute instability in this case, this onset must be represented by some other point on the plots in Fig. 2 where real roots of Eq. (18) merge. We have determined this point with the aid of numerical computations of the critical epsilon and of images of the $L$-contour in the complex $\hat{k}$-plane. A third-order saddle point is present on the real $\hat{k}$-axis when $\epsilon$ has the value corresponding to the inflection point in Fig. $2 \mathrm{~d}$. Increasing $\epsilon$ above this value causes the saddle point to split into two second-order saddle points, each with $\operatorname{Im}(\hat{\omega})=0$. However, if $\epsilon$ is increased sufficiently, these saddle points leave the real $\hat{k}$-axis, and the corresponding frequencies become complex. This behavior can be understood by referring to Fig. 3b. Each of the curves above the inflection point has a minimum to the left and a maximum to the right. For sufficiently large $\hat{\omega}$ these extrema must lie below the inflection point. (See Figs. 2a and 2b.) Consequently, there must be maximum values for the minima and for the maxima in Fig. 3b. As the value of $\epsilon$ is increased above that of either of these maximum extrema, one of the two second-order saddle points leaves the real $\hat{k}$-axis, and its corresponding $\hat{\omega}$ becomes complex. Numerical computations show that the pinch point develops as the value of $\epsilon$ is increased above that of the maximum of the minima of the curves lying above the inflection point in Figs. 2d and $3 \mathrm{~b}$. Therefore, this extreme minimum represents the onset of absolute instability. The condition for a minimum in a curve in Fig. 2 or $3 \mathrm{~b}$ is $\partial \mathcal{D}(\hat{k}, \hat{\omega}) / \partial \hat{k}=0$, and the condition that $\mathcal{D}(\hat{k}, \hat{\omega})$ be a maximum with respect to $\hat{\omega}$ is $\partial \mathcal{D}(\hat{k}, \hat{\omega}) / \partial \hat{\omega}=0$. Solving these equations 
simultaneously and noting that $\hat{k} \neq(\hat{\omega}-s b) / \beta_{\|}$at the mimimum under conderation, we obtain the solutions $\hat{\omega}=\hat{k} / \beta_{\|}$and $\hat{k}=\left(4 b_{0}^{2}\right)^{-1}\left[\beta_{\|} s b \pm\left(\beta_{\|}^{2} s^{2} b^{2}+8 b_{0}^{2} \beta_{\|}^{2}\right)^{\frac{1}{2}}\right]$. We choose the + sign in the latter equation, because this solution is readily shown to correspond to the extreme minimum in Fig. 3b. (The other solution corresponds to the extreme maximum.) The above solutions appear in Eqs. (10) and (11). The critical coupling constant is obtained from Eq. (17). The result is given in Eq. (9).

Finally, we prove that as $s b$ approaches one from below, the critical coupling term $\epsilon_{c}$ approaches zero over the entire range of $\beta_{\|}$in Fig. 1. We first show that in this limit the condition in Eq. (7) or (8) becomes valid for all values of $\beta_{\|}$. Using Eqs. (5) and (6), one can readily show that if $s b \simeq 1$, then the condition in Eq. (8) reduces to $(1-s b)\left(1-1 / 4 \beta_{\|}^{2}\right)+s b>$ 0 . Consequently, in the limit of $s b=1$, the condition in Eq. (8) is valid for all values of $\beta_{\|}$, and Eqs. (4) and (5) can be used to calcutate $\epsilon_{c}$ over the entire range of $\beta_{\|}$. It is evident from Eqs. (4) and (5) that $\epsilon_{c}$ approaches zero as $s b$ approaches one from below. 


\section{DISCUSSION AND CONCLUSIONS}

We have derived exact, analytical conditions for absolute instability in a CRM having a lossless, circular waveguide. The condition for absolute instability is contained in Eqs. (3)(5) over most of the useful range of the CRM parameters. However, in the small $\beta_{\|}$region where the condition in Eq. (8) is not valid, the condition for absolute instabiliy is given by Eqs. (3), (9), and (10). These results are shown graphically in Fig. 1.

The results of the preceding sections, determine only regions of absolute instability in Fig. 1. It is also important to know the conditions under which convective instability is possible. Therefore, we consider whether there is a range of values of $\epsilon$ which gives convective instability for fixed values of the parameters $\beta_{\|}$and $s b$. Numerical computations of $\operatorname{Im}(\hat{\omega})$ for real $\hat{k}$, temporal growth rates of absolute instabilities, and the behavior of $L$-contour images in the complex $\hat{k}$-plane strongly indicate the validity of the following rule. Locate the parameter point on Fig. 1 that corresponds to the onset of absolute instability for the parameters $\beta_{\|}$and $s b$. If this point lies to the right of the curve $A B$, then there exists a range of values of $\epsilon<\epsilon_{c}$ for which convective instability exists. On the other hand, if the parameter point lies to the left of the curve $A B$, then there is no significant range of values of $\epsilon$ for which convective instability is present. That is, for increasing coupling constant, the onsets of instability and of absolute instability occur together. Consequently, unstable couplings of modes whose parameter points lie to the left of $A B$ should be considered as absolutely unstable. [It is proved in Ref. 8 that instability is present for all $\epsilon>0$ if $s b>b_{0}$. Consequently, convective instability is rigorously proved to be possible if $1>s b>b_{0}=\left(1-\beta_{\|}^{2}\right)^{\frac{1}{2}}$. Parameter points $\left(\beta_{\|}, s b\right)$ obeying this condition are located in the lower, right half of the region to the right of $A B$ in Fig. 1.]

This paper has treated only the existence of absolute instabilities in the CRM and not their growth rates. Any system will eventually be disrupted by its fastest-growing absolutely-unstable mode if the system is operated continuously for a sufficient amount of time. However, in a pulsed system there might not be sufficient time for absolute instability to become harmful, provided that the desired convective instability grows sufficiently faster than the fastest growing absolute instability. Consequently, determining whether absolute 
instability will be harmful requires a detailed analysis of growth rates. Finally, we point out that the wavelengths of the absolutely unstable modes should be studied, because the interaction lengths in the experimental devices are finite. Because this analysis has assumed an infinite system in the longitudinal direction, the conditions derived here should be considered as necessary but not sufficient conditions for absolute instability in a device of finite length. 


\section{ACKNOWLEDGEMENTS}

The author wishes to thank B. R. Danly for many helpful discussions. This work was supported in part by the Office of Naval Research. 


\section{References}

1. K. R. Chu and J. L. Hirshfield, Phys. Fluids 21, 461 (1978).

2. V. L. Bratman, N. S. Ginzburg, G. S. Nusinovich, M. I. Petelin, and P. S. Strelkov, Int. J. Electron. 1ㅗ, 541 (1981).

3. A. W. Fliflet, Int. J. Electron. $\underline{61}, 1049$ (1986).

4. K. R. Chu and A. T. Lin, IEEE Trans. Plasma Science 16, 90 (1988).

5. K. D. Pendergast, B. G. Danly, R. J. Tempkin, and J. S. Wurtele, IEEE Trans. on Plasma Science 16, 122 (1988).

6. P. H. Yoon and R.C. Davidson, Phys. Rev. $\underline{\mathbf{A 3 5}}, 2619$ (1987).

7. J. K. Lee, W. D. Bard, S. C. Chiu, R. C. Davidson, and R. R. Goforth, Phys. Fluids 31 , $1824(1988)$.

8. Y. Y. Lau, K. R. Chu, L. R. Barnett, and V. L. Granatstein, Int. J. Infrared and Millimeter Waves $\underline{2}, 373$ (1981).

9. Y. Y. Lau, K. R. Chu, L. R. Barnett, and V. L. Granatstein, Int. J. Infrared and Millimeter Waves $\underline{2}, 395$ (1981).

10. J. A. Davies, R. C. Davidson, and G. L. Johnston, to be published in J. Plasma Phys.

11. R. J. Briggs, Electron Stream Interactions with Plasmas (MIT Press, Cambridge, Mass., 1964).

12. A. Bers, in Basic Plasma Physics 1, edited by A. A. Galeev and R. N. Sudan, Handbook of Plasma Physics, Vol. 1 (North Holland, Amsterdam, 1983), Chap. 3.2.

13. B. G. Danly, K. D. Pendergast, R. J. Temkin, and J. A. Davies, Proc. SPIE $\underline{873}, 143$ (1988). 


\section{Figure Captions}

1. Plot showing parameter regions of absolute instability for the CRM. The coupling of the beam mode and the waveguide mode produces absolute instability if the point $\left(\beta_{\|}, \epsilon\right)$ lies above the curve of $s b$ for the beam mode harmonic.

2. Schematic plots of $\mathcal{D}(\hat{k}, \hat{\omega})$ vs. $\hat{k}$, for fixed $\hat{\omega}$, obtained from Eq. (18) for the case of $s b<b_{0}$ : (a) $\hat{\omega}>1$, (b) $\hat{\omega}=1$, (c) $1>\hat{\omega}>\hat{\omega}_{s}$ [Eq. (6)], (d) $\hat{\omega}=\hat{\omega}_{s}$, (e) $\hat{\omega}_{s}>\hat{\omega}>s b$, (f) $s b>\hat{\omega}>\hat{\omega}_{s-}$, (g) $\hat{\omega}=\hat{\omega}_{s-}$, (h) $\hat{\omega}_{s-}>\hat{\omega}>-1$, (i) $-1>\hat{\omega}$. The quantity $\hat{\omega}_{s-}=4 \beta_{\|} \hat{k}_{s-}+s b$, where $\hat{k}_{s-}$ is obtained by using the negative square root in Eq. (21).

3. Plots of $\mathcal{D}(\hat{k}, \hat{\omega})$ vs. $\hat{k}$, for values of $\hat{\omega}$ shown against the curves, in the neighborhood of the inflection point $\mathrm{P}$ in Fig. 2d: (a) Values of the parameters are $s b=0.6$ and $\beta_{\|}=0.8$. The condition in Eq. (7) is satisfied. The inflection point $P$ lies on the curve $\hat{\omega}=0.98539$. (b) Values of the parameters are $s b=0.6$ and $\beta_{\|}=0.05$. The condition in Eq. (7) is not satisfied. The inflection point $P$ lies on the curve $\hat{\omega}=0.70087$.

4. Plots of $L$-contour images in the complex $\hat{k}$-plane for parameter values of $b=0.6$ and $\beta_{\|}=0.8$. The value of $\epsilon$ at the inflection point P (Fig. 2d) is $\epsilon=0.0036353$. (a) For $\epsilon=0.0035<0.0036353$, there are two saddle points on the real $\hat{k}$-axis. (b) These two saddle points merge into a single third-order saddle point when $\epsilon=0.0036353$. (c) Increasing the value of $\epsilon$ to $\epsilon=0.0038$ produces two saddle points with complex $\hat{k}$ and $\hat{\omega}$. The lower saddle point is a pinch point.

5. Plots of $L$-contour images in the complex $\hat{k}$-plane for parameter values of $b=0.6$ and $\beta_{\|}=0.05$. The value of $\epsilon$ at the inflection point P (Fig. 2d) is $\epsilon=0.004368$. (a) For $\epsilon=0.0035<0.004368$, there are two saddle points with complex $\hat{k}$ and $\hat{\omega}$. Neither is a pinch point. (b) When $\epsilon$ is increased to $\epsilon=0.004368$, these saddle points merge into a single third-order saddle point on the real $\hat{k}$-axis with real $\hat{\omega}$. (c) A further increase in $\epsilon$ to $\epsilon=0.0050$ produces two saddle points on the real $\hat{k}$-axis both with real $\hat{\omega}$. 


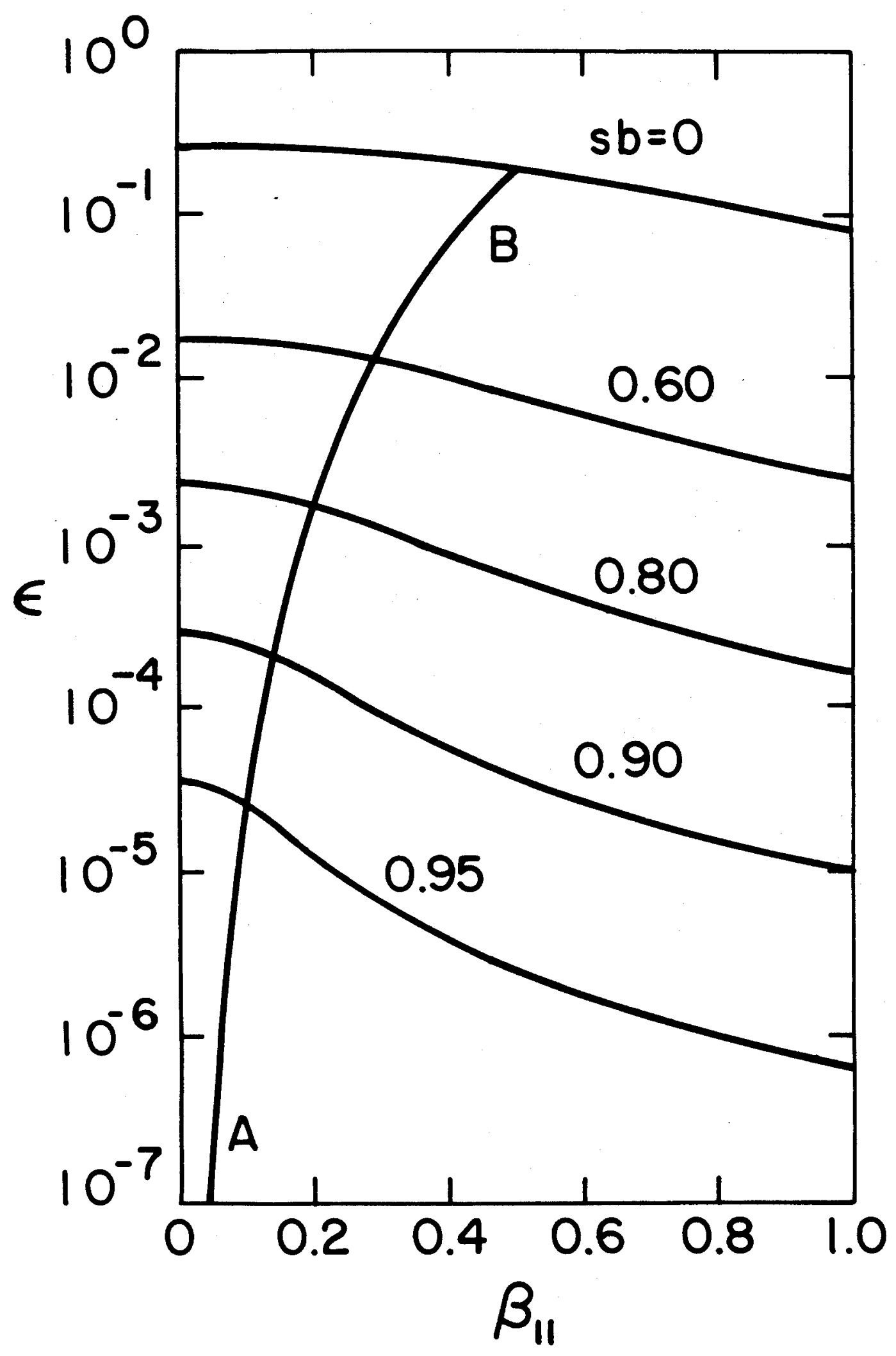

Fig. 1 

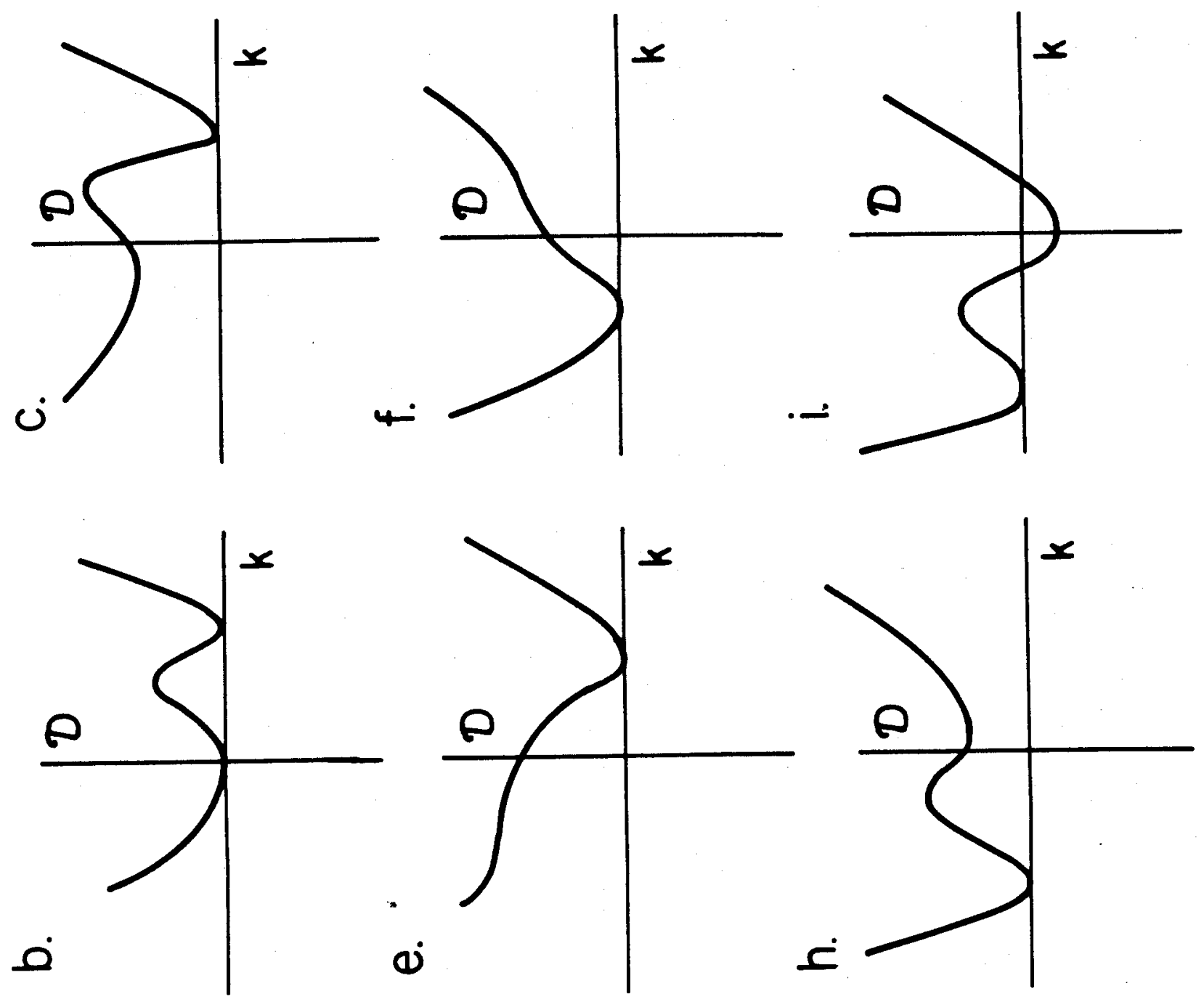

N
iே
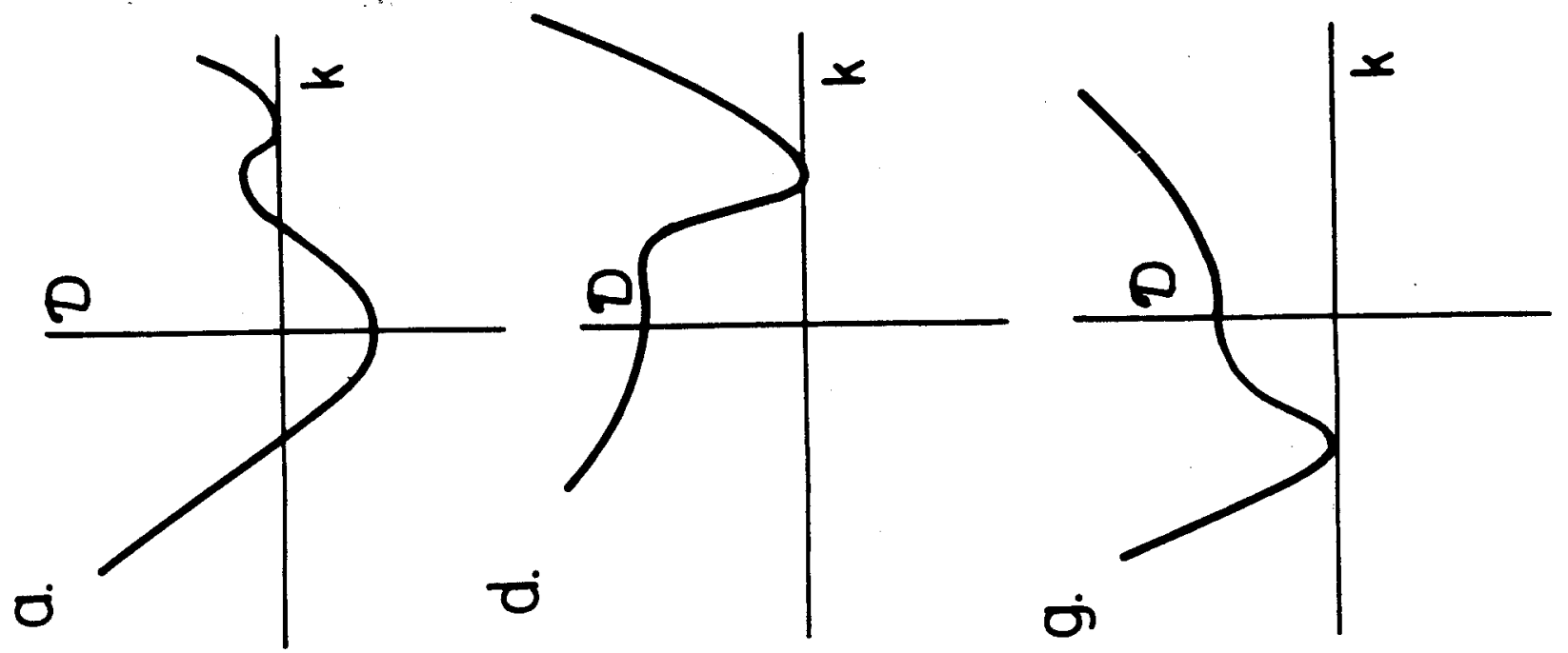


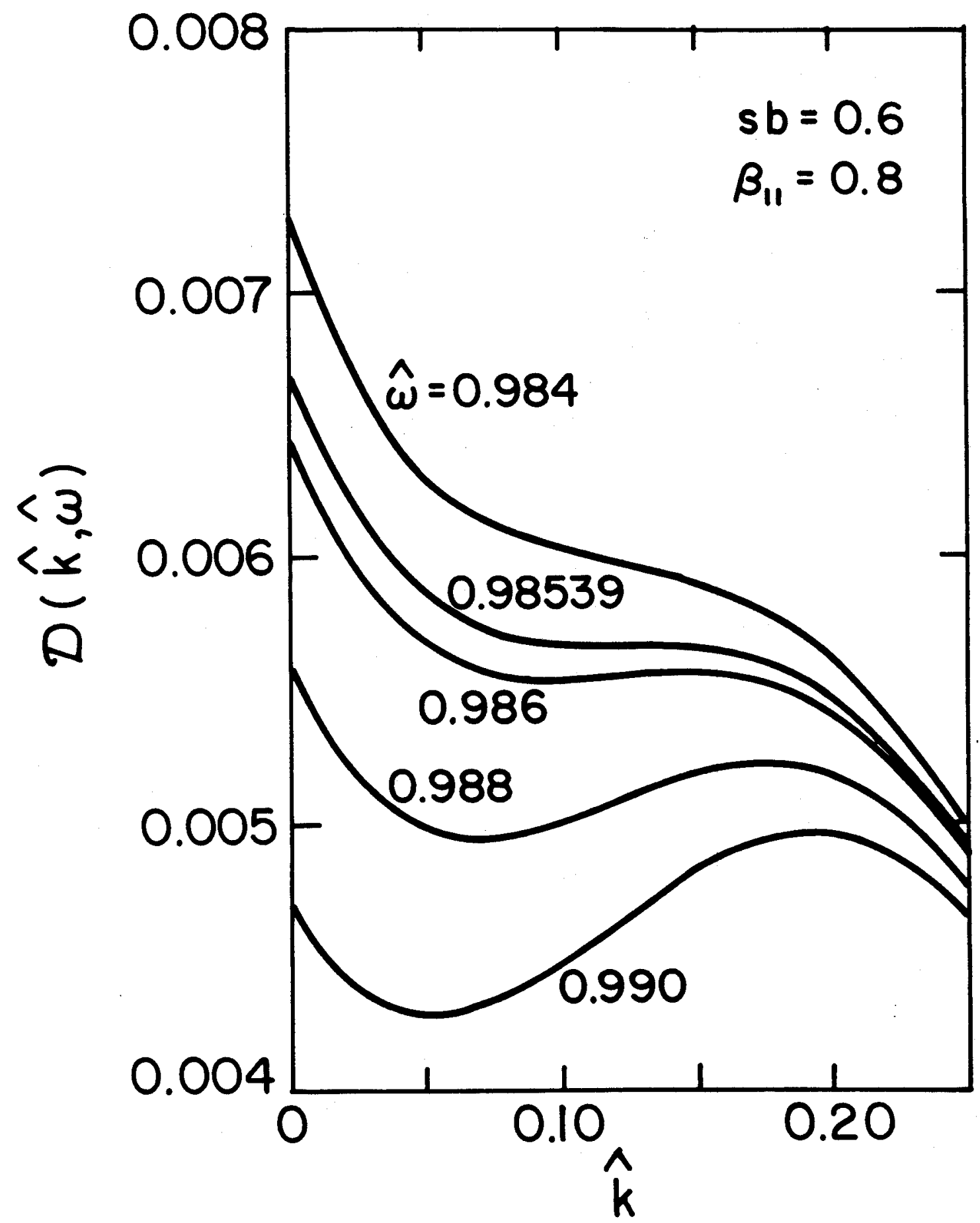

Fig. 3(a) 


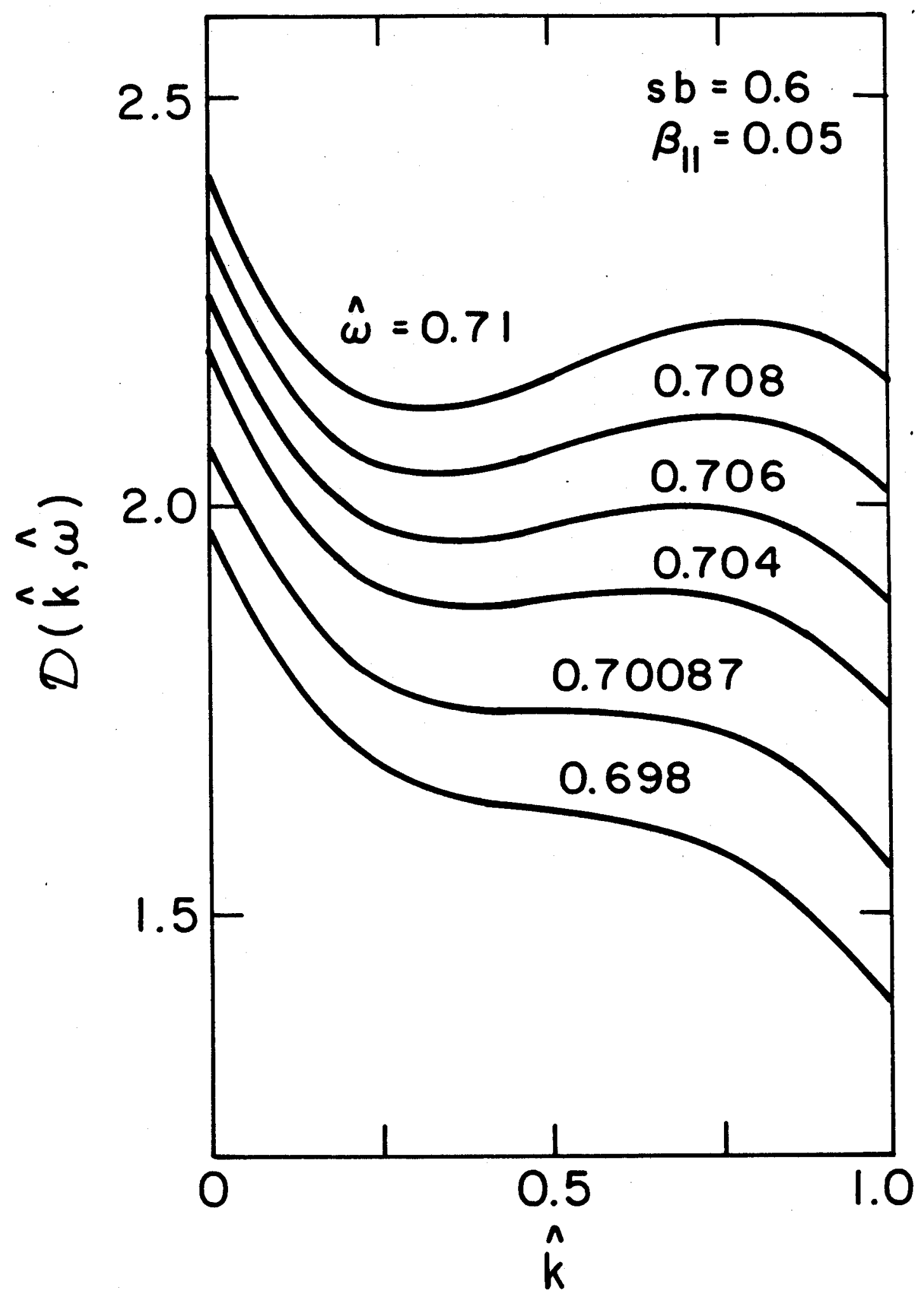

Fig. 3(b) 


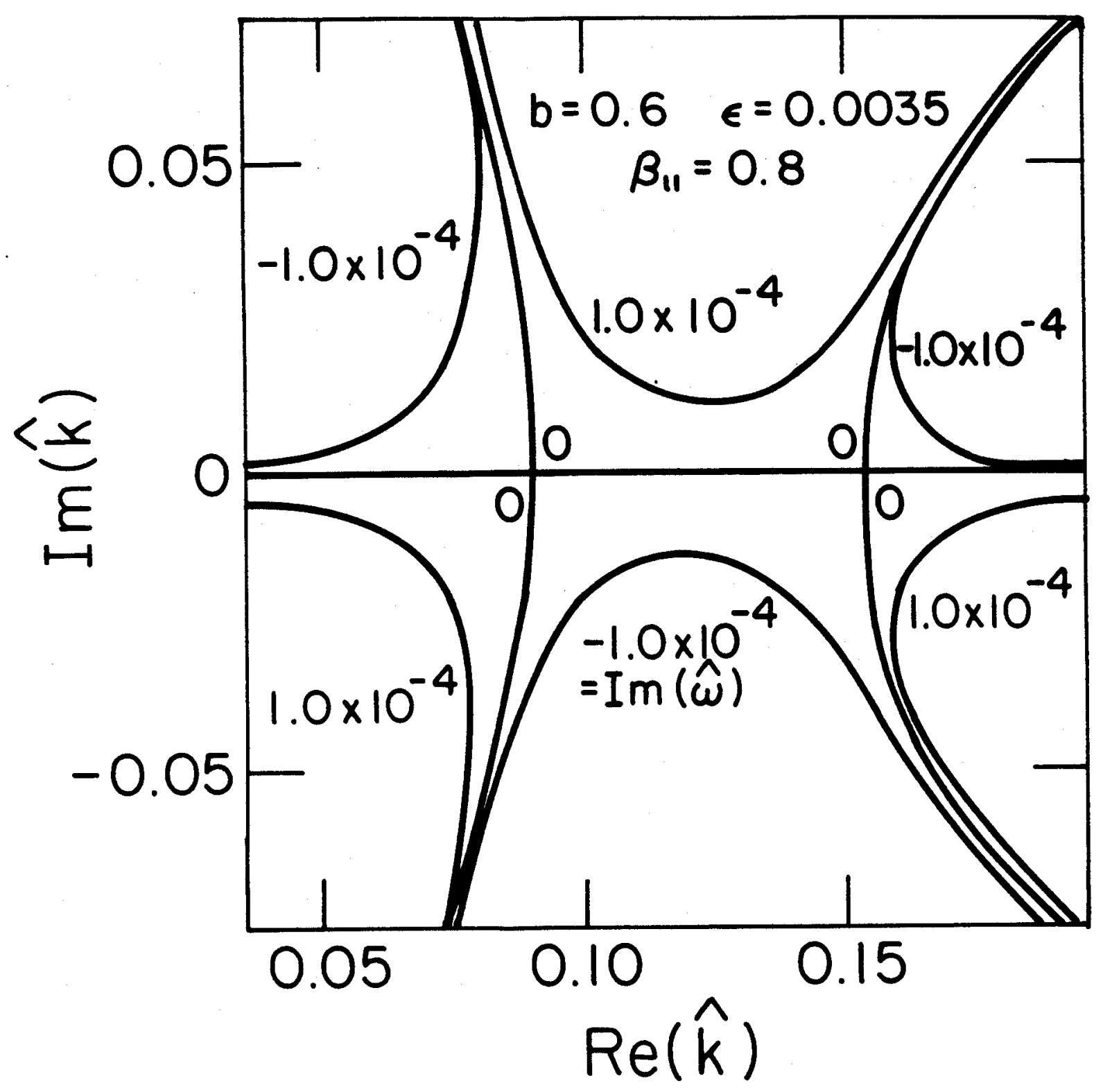

Fig. 4(a) 


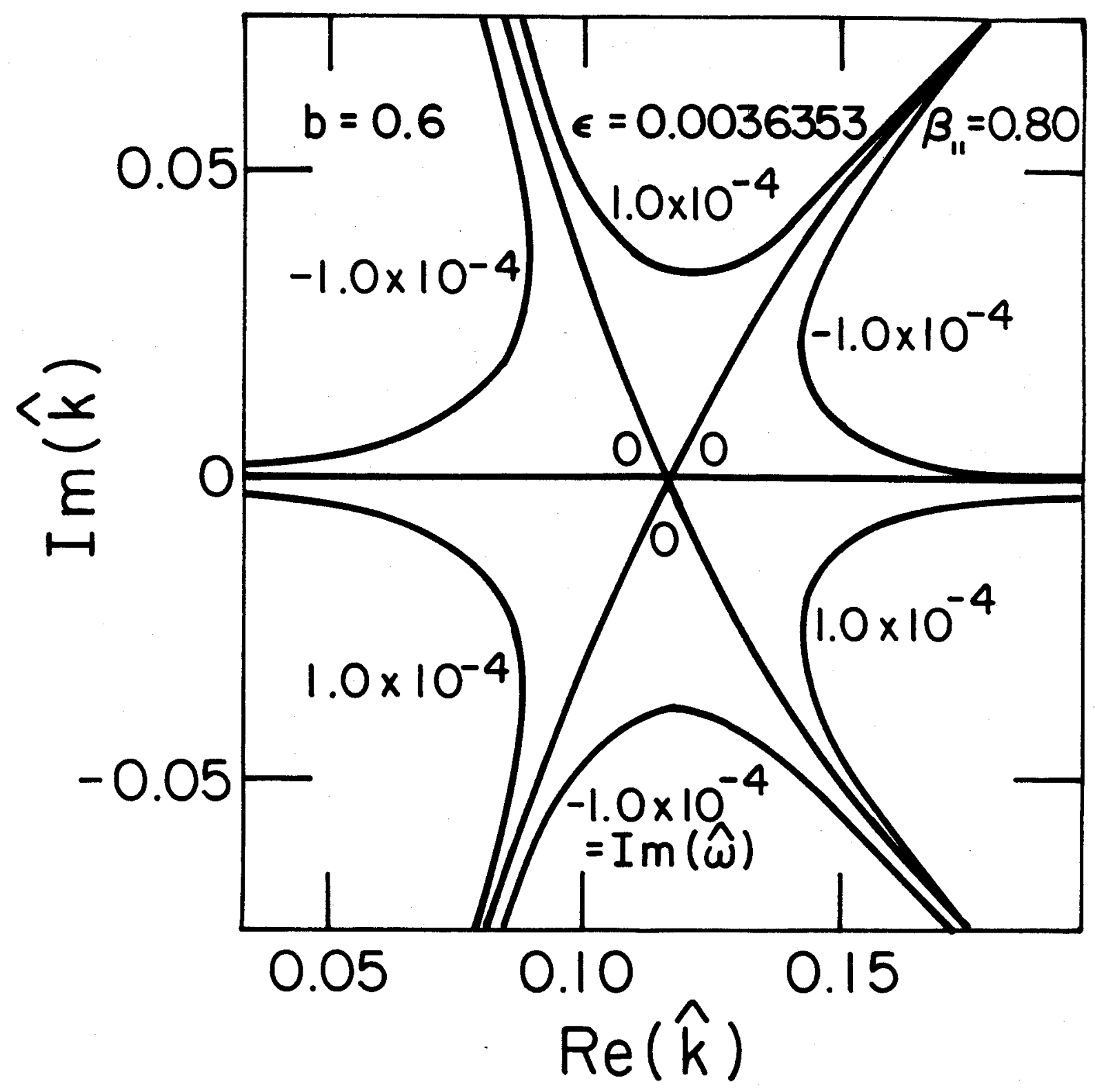

Fig. 4(b) 


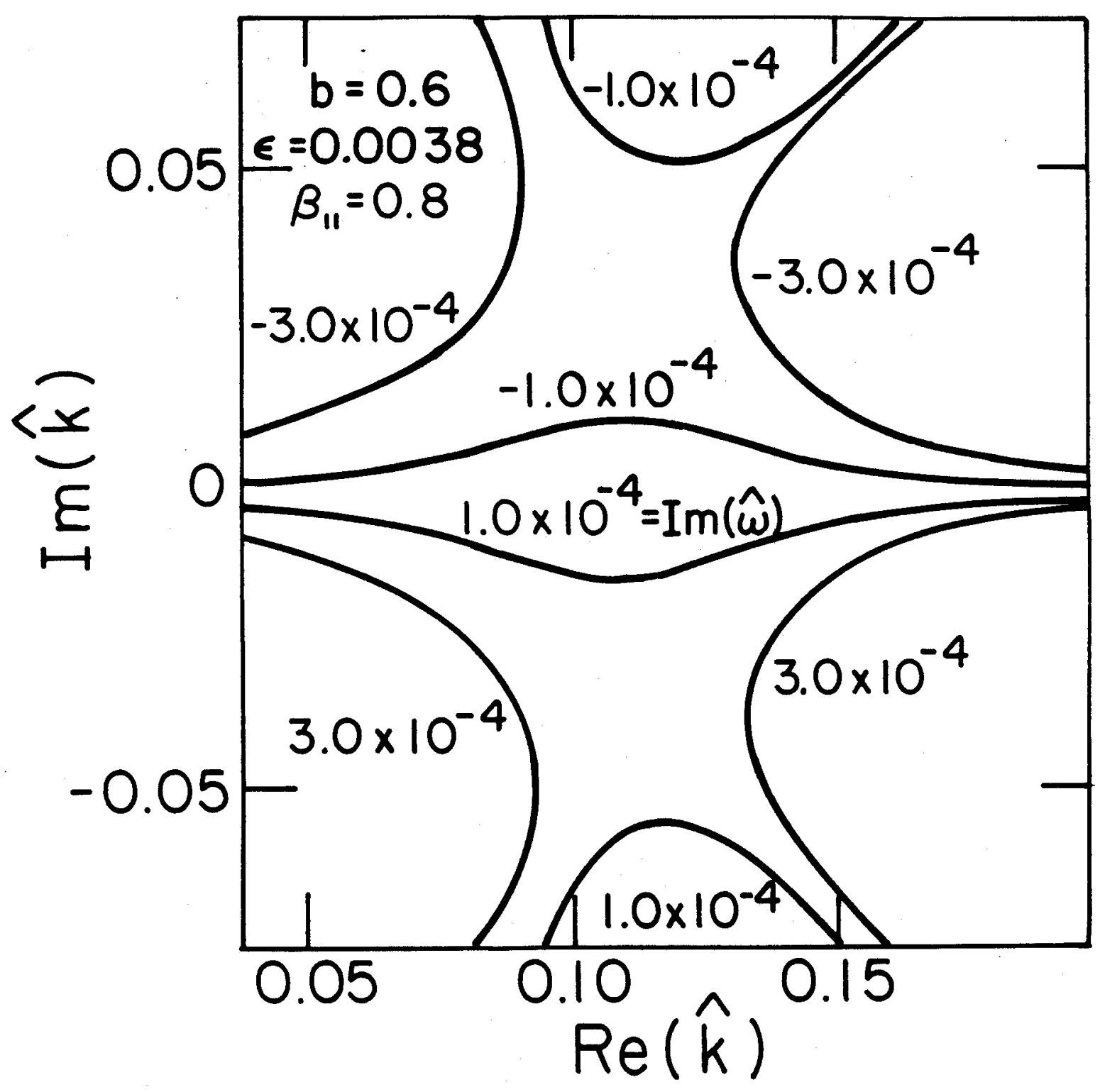

Fig. 4(c) 


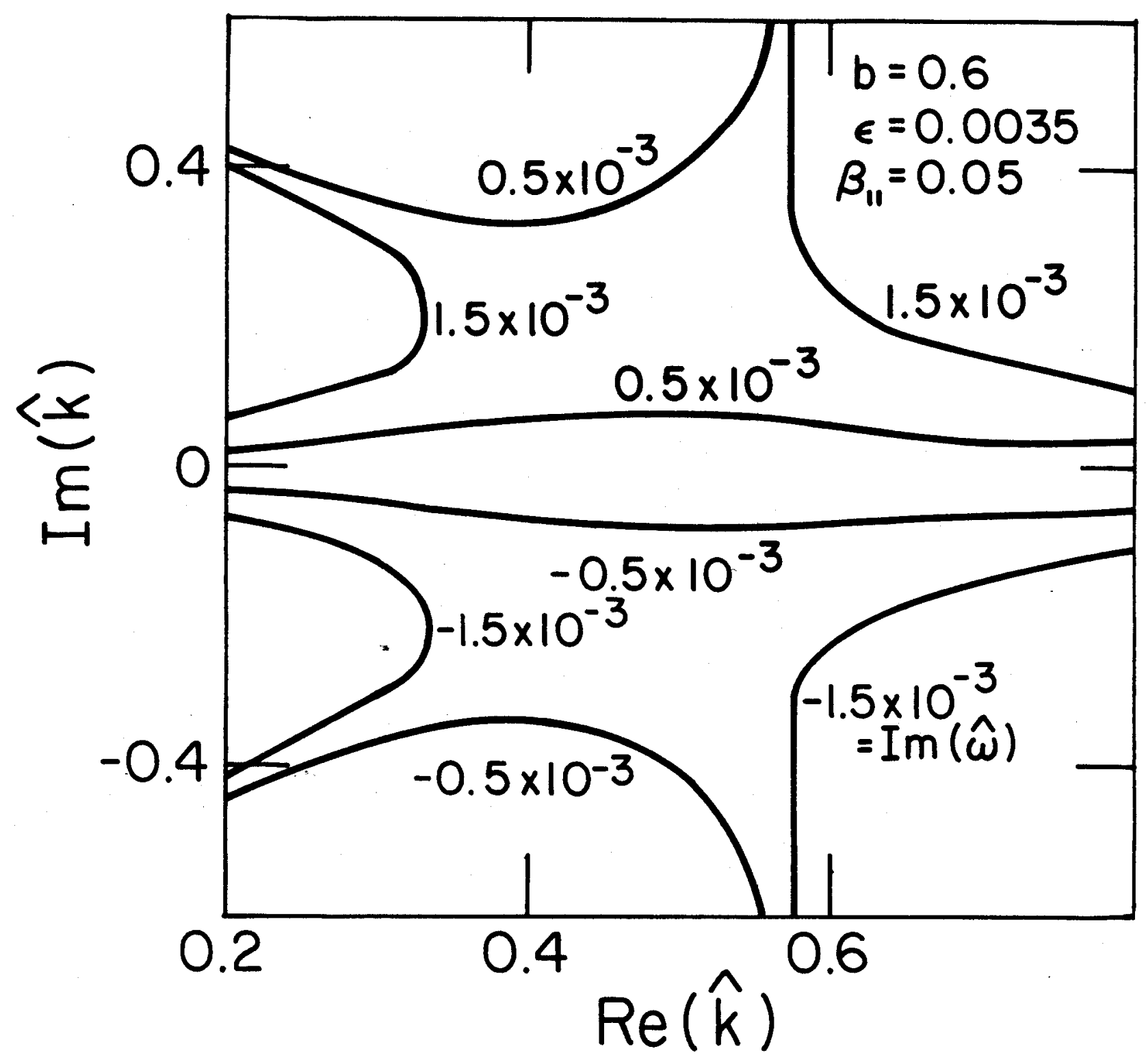

Fig. 5(a) 


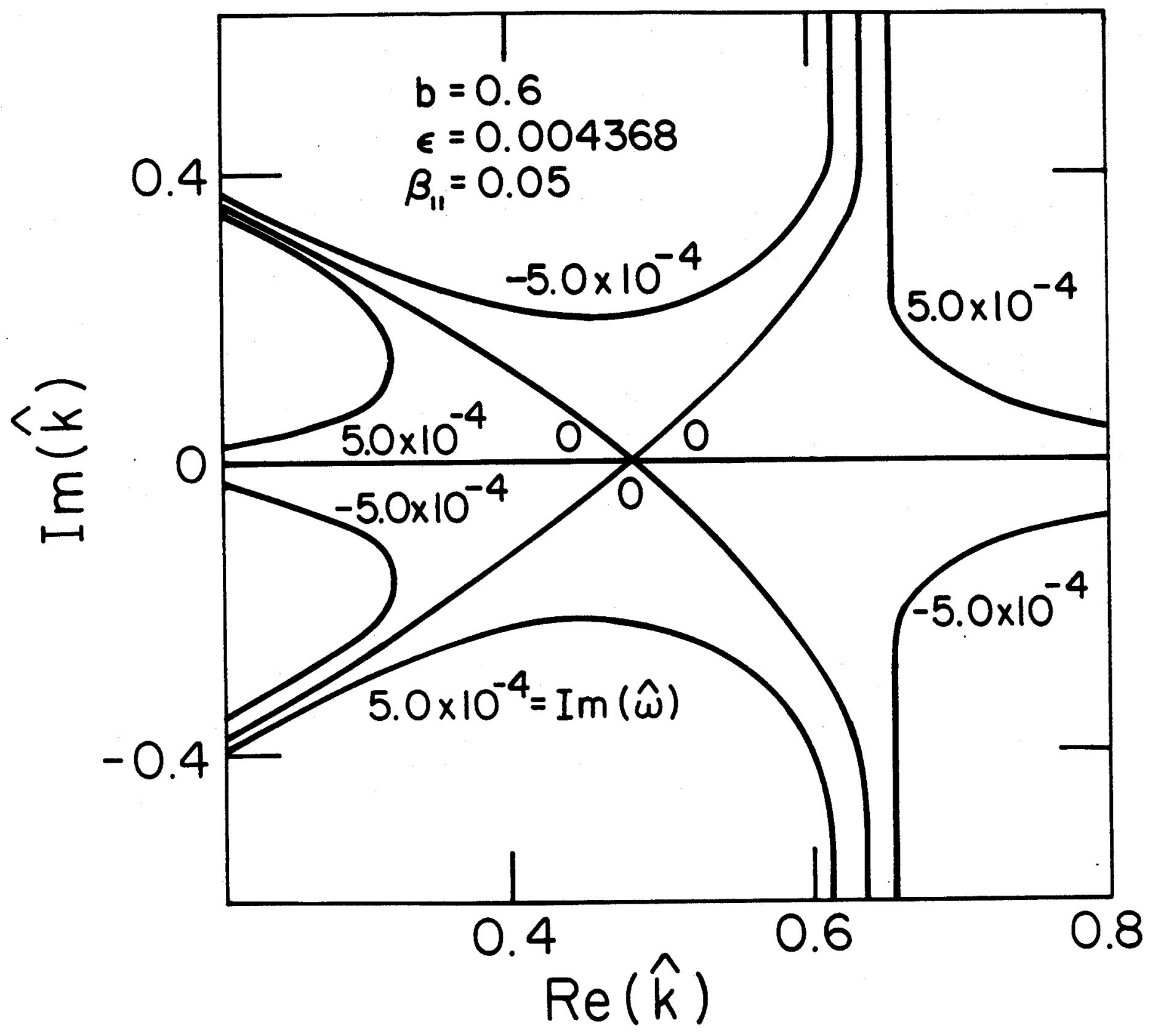

Fig. 5(b) 


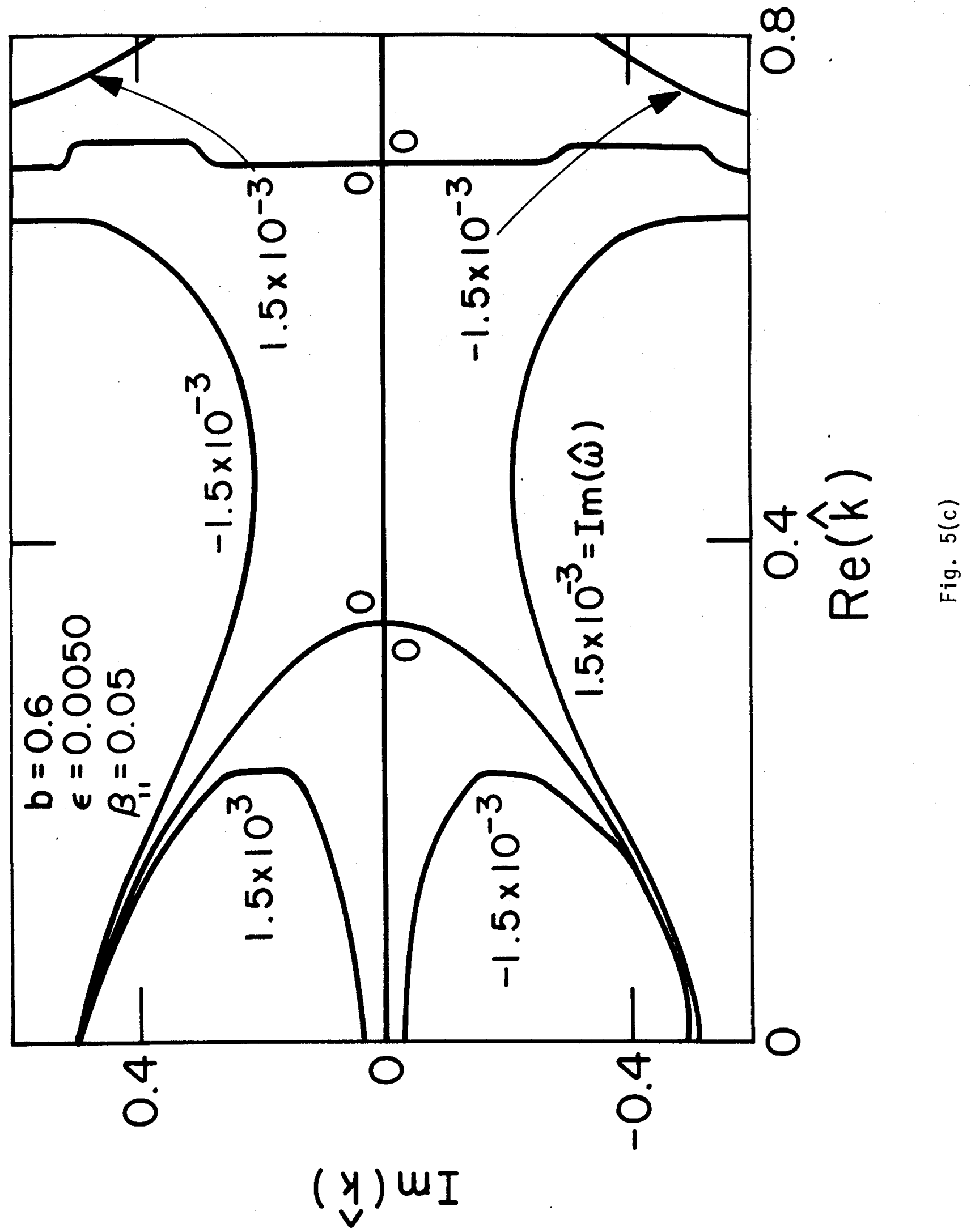

